\title{
Loss of Derivatives in the Infinite Type
}

\author{
Tran Vu Khanh, Stefano Pinton and Giuseppe Zampieri
}

\begin{abstract}
We prove hypoellipticity with loss of $\epsilon$ derivatives for a system of complex vector fields whose Lie-span has a superlogarithmic estimate. In $\mathbb{C} \times R$, the model is $\left(\bar{L}, \bar{f}^{k} L\right)$ where $\bar{f}=\bar{z} h$ for $h \neq 0$ and $L$ is the vector field tangential to the exponentially nondegenerate hypersurface of infinite type defined by $x_{2}=e^{-\frac{1}{|z|^{\alpha}}}$ for $\alpha<1$.
\end{abstract}

Keywords: hypoellipticity, loss of derivatives, superlogarithmic estimate, infinite type.

\section{Introduction}

A system of vector fields $\left(L_{j}\right)_{j}$ has a subelliptic estimate when it has a gain of $\epsilon>0$ derivatives in the sense that $\left\|\Lambda^{\epsilon} u\right\|^{2}<\sum_{j}\left\|L_{j} u\right\|^{2}+\|u\|^{2}, u \in C_{c}^{\infty}$. Here $\Lambda$ is the standard elliptic pseudodifferential operator of order 1. A system which has finite bracket type $2 m$ is a system whose commutators of order $2 m-1$ span the whole tangent space. It is well known that finite type of order $2 m$ implies a $\delta$-subelliptic estimate for some $\epsilon \leq \frac{1}{2 m}$. If $(L, \bar{L})$, in $\mathbb{C} \times \mathbb{R}$, are identified to the generators of the tangential bundle $T^{1,0} M \oplus T^{0,1} M$ to a pseudoconvex hypersurface $M \subset \mathbb{C}^{2}$, then $(L, \bar{L})$ has finite type $2 m$ if and only if the contact of a complex curve $\gamma$ with $M$ is at most $2 m$. Let the hypersurface $M$ be "rigid", that is, graphed by $\operatorname{Re} w=g(z)$ for a real $C^{\infty}$ function $g$, and set $g_{1}=\partial_{z} g, g_{1 \overline{1}}=\partial_{z} \partial_{\bar{z}} g$ and $t=\operatorname{Im} w$. With this notation we have $L=\partial_{z}-i g_{1}(z) \partial_{t}$ and $[L, \bar{L}]=g_{1 \overline{1}} \partial_{t}$. It is assumed that $M$ is pseudoconvex, that is, $g_{1 \overline{1}} \geq 0$ (which also motivates the choice of an even type $2 m$ ). In terms of $g$, the condition of finite type $2 m$ means that $g_{1 \overline{1}}$ has some non-vanishing derivative of order $2(m-1)$. In particular, this happens if $g_{1 \overline{1}} \gtrsim|x|^{2(m-1)}$; in this case, according for example to [12], we have a $\frac{1}{2 m}$-subelliptic estimate.

Received August 12, 2012.

MSC: 32W05, 32W25, 32T25. 
A system has a superlogarithmic estimate if it has logarithmic gain of derivatives with an arbitrarily large constant, that is, for any $\delta$ and for suitable $c_{\delta}$

$$
\|\log (\Lambda) u\|^{2} \underset{\sim}{\sim} \sum_{j}\left\|L_{j} u\right\|^{2}+c_{\delta}\|u\|_{-1}^{2}, \quad u \in C_{c}^{\infty} .
$$

A system which satisfies (1.1) is "precisely $H^{s}$-hypoelliptic" for any $s: u$ is $H^{s}$ where the $L_{j} u$ 's are (Kohn [7]). In particular, the system is $C^{\infty}$-hypoelliptic. Let $L=\partial_{z}-i g_{1}(z) \partial_{t}$ for $g$ of infinite type but exponentially non-degenerate in the sense that for a real curve $S \subset \mathbb{C}$ we have

$$
d_{S}^{\alpha}\left|\log g_{1 \overline{1}}\right| \searrow 0 \text { as } d_{S} \searrow 0 \text { for } \alpha<1
$$

where $d_{S}$ denotes the distance to $S$. Under this assumption, the system $(L, \bar{L})$ has a superlogarithmic estimate (cf. [12]). If we consider the perturbed system $\left(\bar{L}, \bar{f}^{k} L\right)$ for $\bar{f}=\bar{z} h(z)$ with $h \neq 0$ and $k \geq 1$, the system has no more a superlogarithmic estimate, in general; if $k>1$, a logarithmic loss occurs (Proposition 1.4 below). However, it is worth noticing that $\mathcal{L} i e\left(\bar{L}, \bar{f}^{k} L\right)$, the span of commutators of order $\leq k-1$, has a superlogarithmic estimate (since it produces $L$ as a commutator of order $k-1$ ). We are able to prove below that, in the terminology of Kohn [8], the system $\left(\bar{L}, \bar{f}^{k} L\right)$ has an arbitrarily small loss of $\epsilon$ derivatives and thus, in particular, is $C^{\infty}$, but not exactly $H^{s}$-, hypoelliptic. Let $\zeta_{0}$ and $\zeta_{1}$ be cut-off functions in a neighborhood of 0 with $\zeta_{0} \prec \zeta_{1}$ in the sense that $\zeta_{1} \equiv 1$ over a neighborhood of $\operatorname{supp} \zeta_{0}$.

Theorem 1.1. Let $L=\partial_{z}-i g_{1}(z) \partial_{t}$ and assume that 0 is a point of infinite type, i.e. $g_{1 \overline{1}}=0^{\infty}(|z|)$ but not exponentially degenerate, i.e. (1.2) is fulfilled. Then the system $\left(\bar{L}, \bar{f}^{k} L\right)$ (any $k$ ) has an arbitrarily small loss of $\epsilon$ derivatives, that is,

$$
\left\|\zeta_{0} u\right\|_{s}^{2} \lesssim\left\|\zeta_{1} \bar{L} u\right\|_{s+\epsilon}^{2}+\left\|\zeta_{1} \bar{f}^{k} L u\right\|_{s+\epsilon}^{2}+\left\|\bar{f}^{k} u\right\|_{\epsilon}^{2}+\|u\|_{0}^{2}
$$

The proof of this theorem and of the two propositions below follows in Section 2. Generally, an estimate of type (1.3) for smooth $u$ does not yield finiteness of $\left\|\zeta_{0} u\right\|_{s}$ for a $H^{\epsilon}$-solution $u$ of $\bar{L} u=f, \bar{f}^{k} L u=g$ when $\zeta_{1} f$ and $\zeta_{1} g$ are in $H^{s+\epsilon}$. However, $L$ has coefficient $t$-independent and therefore it commutes with the $t$-derivatives. On the other hand, the $t$-derivatives describe the full Sobolev norm on the "positively microlocalized" component $u^{+}$(cf. $\S 2$ below) which is the only one which needs to be controlled. For 
this reason, if we use a sequence of pseudodifferential smoothing operators in $t, \chi_{\nu}\left(\partial_{t}\right) \rightarrow$ id as in [8] and [1], and we remark that

$$
\bar{L}\left(\chi_{\nu}\left(\partial_{t}\right) u^{+}\right)=\chi_{\nu}\left(\partial_{t}\right)\left(\bar{L} u^{+}\right)+\text {Order }_{-\infty},
$$

then, (1.3) applied to $\Lambda^{s}\left(\chi_{\nu}\left(\partial_{t}\right) u^{+}\right)=\chi_{\nu}\left(\partial_{t}\right)\left(\Lambda^{s} u^{+}\right)$yields

Corollary 1.2. In the situation of Theorem 1.1, the system $\left(\bar{L}, \bar{f}^{k} L u\right)$ is hypoelliptic with loss of $\epsilon$ derivatives: $\left(\bar{L} u, \bar{f}^{k} L u\right) \in H^{s+\epsilon}, u \in H^{\epsilon}$ implies $u \in H^{s}$.

For $k=1$ we have an estimate for local regularity without loss

Proposition 1.3. In the situation above, assume in addition

$$
\left|g_{1}\right| \lesssim g_{1 \overline{1}}^{\frac{1}{2}}
$$

then

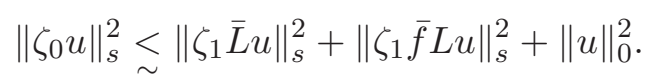

When $k>1$, a loss must occur

Proposition 1.4. Assume that $g=e^{-\frac{1}{|z|^{\alpha}}}$. If

$(1 . \emptyset) \zeta_{0} u\left\|_{s}^{2}<\right\|(\log \Lambda)^{r} \zeta_{1} \bar{L} u\left\|_{s}^{2}+\right\|(\log \Lambda)^{r} \zeta_{1} \bar{f}^{k} L u\left\|_{s}^{2}+\right\| \bar{f}^{k} u\left\|_{\epsilon}^{2}+\right\| u \|_{0}^{2}$,

then we must have $r \geq \frac{k-(\alpha+1)}{\alpha}$.

As far as we know, this is the first time that the problem of hypoellipticity is discussed for degenerate vector fields $\left(\bar{L}, \bar{f}^{k} L\right)$ obtained from $L=\partial_{z}-i g_{1}(z) \partial_{t}$ of infinite type, that is, satisfying $g_{1 \overline{1}}=0^{\infty}(|z|)$. However, it is necessary to make further assumptions such as (1.2). This guarantees a superlogarithmic estimate ([12]), and in turn, hypoellipticity according to Kohn [7]. Hypoellipticity with loss of derivatives for $L=\partial_{z}-i \bar{z} \partial_{t}$ was discovered by Kohn in [8]. In this case, $L$ is the $(1,0)$ vector field tangential to the strictly pseudoconvex hypersurface $\operatorname{Re} w=|z|^{2}$ and the loss amounts to $\frac{k-1}{2}$. The problem was further discussed by Bove, Derridj, Kohn and Tartakoff in [1] essentially for the vector field $L=\partial_{z}-i \bar{z}|z|^{2(m-1)} \partial_{t}$ tangential to the hypersurface $\operatorname{Re} w=|z|^{2 m}$ and the resulting loss is $\frac{k-1}{2 m}$. In both cases 
the conclusion extends to the sum of squares $L \bar{L}+\bar{L}|z|^{2 k} L$ and the loss doubles to $\frac{k-1}{m}$. Moreover, in [1], analytic hypoellipticity has been proved; notice that this cannot be discussed in our framework, since, $g$ having infinite type, it cannot be real analytic. For the vector fields $L=\partial_{z}-i g_{1}(z) \partial_{t}$ tangential to a general pseudoconvex hypersurface of finite type with $g_{1 \overline{1}}$ vanishing at order $2(m-1)$ along a real curve), hypoellipticity with loss of $\frac{k-1}{2 m}$ derivatives has been proved by the authors in [11]. Under some additional conditions, the result also extends to sums of squares (with double loss $\frac{k-1}{m}$ ). When the hypersurface has infinite type as in the present paper, it is therefore natural to expect an arbitrarily small loss of derivatives.

\section{Technical preliminaries and Proof}

Our ambient space is $\mathbb{C} \times \mathbb{R}$ identified with $\mathbb{R}^{3}$ endowed with coordinates $(z, \bar{z}, t)$ or $(\operatorname{Re} z, \operatorname{Im} z, t)$. We denote by $\xi=\left(\xi_{\operatorname{Re} z}, \xi_{\operatorname{lm} z}, \xi_{t}\right)$ the variables dual to $(\operatorname{Re} z, \operatorname{Im} z, t)$, by $\Lambda_{\xi}^{s}$ the standard symbol $\left(1+|\xi|^{2}\right)^{\frac{s}{2}}$, and by $\Lambda^{s}$ the pseudodifferential operator with symbol $\Lambda_{\xi}^{s}$; this is defined by $\Lambda^{s}(u)=\mathcal{F}^{-1}\left(\Lambda_{\xi}^{s} \mathcal{F}(u)\right)$ where $\mathcal{F}$ is the Fourier transform. We also consider the partial symbol $\Lambda_{\xi_{t}}^{s}$ and the associate pseudodifferential operator $\Lambda_{t}^{s}$. We denote by $\|u\|_{s}:=\left\|\Lambda^{s} u\right\|_{0}$ (resp. $\|u\|_{\mathbb{R}, s}:=\left\|\Lambda_{t}^{s} u\right\|_{0}$ ) the full (resp. totally real) $s$-Sobolev norm. We use the notation $\underset{\sim}{\sim}$ and $\underset{\sim}{<}$ to denote inequalities up to multiplicative constants; we denote $\underset{\mathrm{ry}}{\sim} \sim$ the combination of $>$ and $\underset{\sim}{\sim}$ In $\mathbb{R}_{\xi}^{3}$, we consider a conical partition of the unity $1=\psi^{+}+\stackrel{\sim}{\sim}+\psi^{0}$ where $\psi^{ \pm}$have support in a neighborhood of the axes $\pm \xi_{t}$ and $\psi^{0}$ in a neighborhood of the plane $\xi_{t}=0$, and introduce a decomposition of the identity id $=\Psi^{+}+\Psi^{-}+\Psi^{0}$ by means of $\Psi^{\frac{ \pm}{0}}$, the pseudodifferential operators with symbols $\psi^{\frac{ \pm}{0}}$; we accordingly write $u=u^{+}+u^{-}+u^{0}$. Since $\left|\xi_{\operatorname{Re} z}\right|+\left|\xi_{\operatorname{lm} z}\right| \lesssim \xi_{t}$ over $\operatorname{supp} \psi^{+}$, then $\left\|u^{+}\right\|_{\mathbb{R}, s} \sim\left\|u^{+}\right\|_{s}$.

We carry on the discussion by describing the properties of commutation of the vector fields $L$ and $\bar{L}$ for $L=\partial_{z}-i g_{1}(z) \partial_{t}$. The crucial equality is

$$
\|L u\|^{2}=([L, \bar{L}] u, u)+\|\bar{L} u\|^{2}, \quad u \in C_{c}^{\infty},
$$

which is readily verified by integration by parts. Since $\sigma\left(\partial_{t}\right)$, the symbol of $\partial_{t}$, is dominated by $\sigma(L)$ and $\sigma(\bar{L})$ in the "elliptic region" (the support of $\psi^{0}$ ) and since $L$ can be controlled by $\bar{L}$ with an additional $\epsilon \partial_{t}$ (because of $(2.1))$, then $\left\|u^{0}\right\|_{1}^{2} \lesssim\|\bar{L} u\|_{0}^{2}+\|u\|_{0}^{2}$. As for $u^{-}$, recall that $[L, \bar{L}]=g_{1 \overline{1}} \partial_{t}$ 
and hence $g_{1 \overline{1}} \sigma\left(\partial_{t}\right) \leq 0$ over $\operatorname{supp} \psi^{-}$. Thus (2.1) yields $\|L u\|^{2}<\|\bar{L} u\|^{2}$. It follows that, if $L$ and $\bar{L}$ have superlogarithmic estimate as in our application, then

$$
\left\|\log (\Lambda) u^{-}\right\|^{2} \leq \delta\left\|\bar{L} u^{-}\right\|^{2}+c_{\delta}\|u\|^{2} .
$$

In conclusion, only estimating $u^{+}$is relevant. We note here that, over $\operatorname{supp} \Psi^{+}$, we have $g_{1 \overline{1}} \xi_{t} \geq 0$; thus

$$
\begin{aligned}
\left\|g_{1 \overline{1}}^{\frac{1}{2}} u^{+}\right\|_{\frac{1}{2}}^{2} & =\left|\left([L, \bar{L}] u^{+}, u^{+}\right)\right| \\
& \leq\left\|L u^{+}\right\|^{2}+\left\|\bar{L} u^{+}\right\|^{2} .
\end{aligned}
$$

Following Kohn [7], we introduce a microlocal modification of $\Lambda^{s}$, denoted by $R^{s}$; this is the pseudodifferential operator with symbol $R_{\xi}^{s}:=$ $\left(1+|\xi|^{2}\right)^{\frac{s \sigma(x)}{2}}, \sigma \in C_{c}^{\infty}$, that is, $R^{s}(u)=\mathcal{F}^{-1}\left(R_{\xi}^{s} \mathcal{F}(u)\right)$. Often, what is used is in fact the partial operator in $t$ with symbol $R_{\xi_{t}}^{s}$. We denote it by the same symbol $R^{s}$ and observe that, $f$ being independent of $t$, we have

$$
\left[R^{s}, f\right]=0 \text {. }
$$

The relevant property of $R^{s}$ is

$$
\left\|\Lambda^{s} \zeta_{0} u\right\|^{2}<\left\|R^{s} \zeta_{0} u\right\|^{2}+\left\|\zeta_{0} u\right\|^{2} \quad \text { if } \zeta_{0} \prec \sigma .
$$

Thus, $R^{s}$ is equivalent to $\Lambda^{s}$ over functions supported in the region where $\sigma \equiv 1$. In addition, $\zeta_{1} R^{s}$ better behaves with respect to commutation with $L$; in fact, Jacobi equality yields

$$
\left[\zeta_{1} R^{s}, L\right] \sim \dot{\zeta}_{1} R^{s}+\zeta_{1} \log (\Lambda) R^{s}
$$

Thus, on one hand we have the disadvantage of the additional $\log (\Lambda)$ in the second term, but we gain much in the cut-off because

$$
\dot{\zeta}_{1} R^{s} \text { is of order } 0 \text { if } \operatorname{supp} \dot{\zeta}_{1} \cap \operatorname{supp} \sigma=\emptyset .
$$

Property (2.5) is crucial in localizing regularity in presence of superlogarithmic estimate.

Proof of Theorem 1.1. As it has already been noticed, it suffices to prove (1.3) only for $u^{+}$and for $\|\cdot\|_{\mathbb{R}, s}$; thus we write for simplicity $u$ and $\|\cdot\|_{s}$ but mean $u^{+}$and $\|\cdot\|_{\mathbb{R}, s}$. Moreover, we can use a cut-off $\zeta=\zeta(t)$ in $t$ only. In fact, for a cut-off $\zeta=\zeta(z)$ we have $[L, \zeta(z)]=\dot{\zeta}$ and $\dot{\zeta} \equiv 0$ at $z=0$. On the 
other hand, $\bar{f}^{k} L \sim L$ outside $z=0$ which yields gain of derivatives, instead of loss. We call "good" a term in the right side (upper bound) of an estimate we wish to prove and "absorbable" a term which comes as a fraction (small constant or sc) of a formerly encountered term. We take cut-off functions in a neighborhood of 0 : $\zeta_{0} \prec \sigma \prec \zeta_{1}$; we have for $u \in C^{\infty}$

$$
\begin{aligned}
\left\|\zeta_{0} u\right\|_{s}^{2} & =\left\|\zeta_{0} \zeta_{1} u\right\|_{s}^{2} \\
& <\left\|R^{s} \zeta_{0} \zeta_{1} u\right\|^{2}+\|u\|_{0}^{2} \\
& <\left\|\zeta_{0} R^{s} \zeta_{1} u\right\|_{0}^{2}+\left\|\left[R^{s}, \zeta_{0}\right] \zeta_{1} u\right\|_{0}^{2}+\|u\|_{0}^{2} \\
& <\left\|R^{s} \zeta_{1} u\right\|_{0}^{2}+\|u\|_{0}^{2} \\
& \sim\left\|\zeta_{1} R^{s} \zeta_{1} u\right\|_{0}^{2}+\|u\|_{0}^{2}, \\
& \sim \|{ }^{2}
\end{aligned}
$$

where the inequality in the fourth line follows from interpolation in Sobolev spaces and the last from $\operatorname{supp}\left(1-\zeta_{1}\right) \cap \operatorname{supp} \sigma=\emptyset$. We have

$$
\begin{aligned}
\left\|\zeta_{0} u\right\|_{s}^{2} & \underset{\text { by } \underset{\sim}{\sim}}{<} \underbrace{\left\|\zeta_{1} R^{s} \zeta_{1} u\right\|^{2}}_{(a)}+\|u\|^{2} \\
& \leq \delta\left(\left\|L\left(\zeta_{1} R^{s} \zeta_{1}\right) u\right\|^{2}+\left\|\bar{L}\left(\zeta_{1} R^{s} \zeta_{1}\right) u\right\|^{2}\right)+c_{\delta}\|u\|^{2}
\end{aligned}
$$

where the inequality marked by $(*)$ follows from compactness which is a byproduct of superlogarithmic estimate. In the last line, we leave aside the central term and attack the first. Using integration by parts, we have

$$
L \underset{\sim}{\sim}+[L, \bar{L}] \quad \text { microlocally on } \operatorname{supp} \psi^{+}
$$

We rewrite the commutator. For this we recall an easy result about interpolation in Sobolev spaces. For positive $\epsilon, r, n_{1}, n_{2}$ with $n_{1}$ and $n_{2}$ integers satisfying $0<n_{1} \leq r$ and $n_{2}>0$,

$$
\left\|h^{r} u\right\|_{\frac{1}{2}}^{2} \leq s c\left\|h^{r-n_{1}} u\right\|_{\frac{1}{2}-n_{1} \epsilon}^{2}+l c\left\|h^{r+n_{2}} u\right\|_{\frac{1}{2}+n_{2} \epsilon}^{2} .
$$

We apply $(2.9)$ for $h=g_{1 \overline{1}}^{\frac{1}{2 r}}, n_{1}=r, \epsilon=\frac{1}{2 r}, n_{2}=1$ (and note that $h$ needs not to be smooth because $h$ is a function of $z$ whereas Sobolev norms are taken with respect to $t$ ). We observe that, since $g$ has infinite order, then 
$g_{1 \overline{1}}^{\frac{1}{2 r}} \lesssim|f|^{k}$ for any $r$ and any $k$. It follows

$$
\begin{aligned}
\left.\|\|[L, \bar{L}]\right|^{\frac{1}{2}} \zeta_{1} R^{s} \zeta_{1} u \|^{2} & =\left\|g_{1 \overline{1}}^{\frac{1}{2}} \zeta_{1} R^{s} \zeta_{1} u\right\|_{\frac{1}{2}}^{2} \\
& <\mathrm{sc}\left\|\zeta_{1} R^{s} \zeta_{1} u\right\|_{0}^{2}+\mathrm{lc}\left\|g_{1 \overline{1}}^{\frac{1}{2}} g_{1 \overline{1}}^{\frac{1}{2 r}} \zeta_{1} R^{s} \zeta_{1} u\right\|_{\frac{1}{2}+\frac{1}{2 r}}^{2} \\
& <\mathrm{sc}\left\|\zeta_{1} R^{s} \zeta_{1} u\right\|_{0}^{2}+\mathrm{lc}\left\|g_{1 \overline{1}}^{\frac{1}{2}} \Lambda^{\frac{1}{2}} \bar{f}^{k} \zeta_{1} R^{s} \zeta_{1} u\right\|_{\epsilon}^{2} \\
& =\operatorname{sc}\left\|\zeta_{1} R^{s} \zeta_{1} u\right\|_{0}^{2}+\mathrm{lc}\left\|[L, \bar{L}]^{\frac{1}{2}} \bar{f}^{k} \zeta_{1} R^{s} \zeta_{1} u\right\|_{\epsilon}^{2} \\
& <\left\|\zeta_{1} R^{s} \zeta_{1} u\right\|_{0}^{2}+\left\|L \bar{f}^{k}\left(\zeta_{1} R^{s} \zeta_{1}\right) u\right\|_{\epsilon}^{2}+\left\|\bar{L} \bar{f}^{k}\left(\zeta_{1} R^{s} \zeta_{1}\right) u\right\|_{\epsilon}^{2} \\
& \sim
\end{aligned}
$$

We wish to first estimate the second term in the bottom of (2.10) in which we also replace $L \bar{f}^{k}$ by $\bar{f}^{k} L$. In doing so, we encounter an error term $\left\|\bar{f}^{k}\left(\zeta_{1} R^{s} \zeta_{1}\right) u\right\|_{\epsilon}^{2}$ that we will estimate later on; (in fact, $\left[L, \bar{f}^{k}\right] \sim \bar{f}^{k}$ since $\bar{f}=\bar{z} h$ and $[L, \bar{z}]=0)$. After this, we recall Jacobi identity, observe that $\left[\bar{f}^{k} L, \zeta_{1} R^{s} \zeta_{1}\right]$ has order arbitrarily close to $s-1$ (because of a logarithmic extra term), that is

$$
\left[\bar{f}^{k} L, \zeta_{1} R^{s} \zeta_{1}\right]=\left[L, \zeta_{1}\right] R^{s} \zeta_{1} \bar{f}^{k}+\zeta_{1}\left[\bar{L}, R^{s}\right] \zeta_{1} \bar{f}^{k}+\zeta_{1} R^{s}\left[\bar{L}, \zeta_{1}\right] \bar{f}^{k}
$$

$$
\sim \underbrace{\dot{\zeta}_{1} R^{s} \zeta_{1}}_{\text {0-order by }(2.5)} \bar{f}^{k}+\underbrace{\zeta_{1} \log (\Lambda) R^{s} \zeta_{1}}_{\text {by }(2.4)} \bar{f}^{k}+\underbrace{\zeta_{1} R^{s} \dot{\zeta}_{1}}_{0 \text {-order by }(2.5)} \bar{f}^{k} .
$$

Thus we can commutate $\bar{f}^{k} L$ with $\zeta_{1} R^{s} \zeta_{1}$ up to an error as described in (2.11) which yields

$$
\begin{aligned}
\left\|L \bar{f}^{k}\left(\zeta_{1} R^{s} \zeta_{1}\right) u\right\|_{\epsilon}^{2} & <\left\|\bar{f}^{k} L\left(\zeta_{1} R^{s} \zeta_{1}\right) u\right\|_{\epsilon}^{2}+\left\|\bar{f}^{k}\left(\zeta_{1} R^{s} \zeta_{1}\right) u\right\|_{\epsilon}^{2} \\
& <\left\|\left(\zeta_{1} R^{s} \zeta_{1}\right) \bar{f}^{k} L u\right\|_{\epsilon}^{2}+\left\|\left(\zeta_{1} \log (\Lambda) R^{s} \zeta_{1}\right) \bar{f}^{k} u\right\|_{\epsilon}^{2} \\
& +\left\|\bar{f}^{k} u\right\|_{\epsilon}^{2}+\left\|\bar{f}^{k}\left(\zeta_{1} R^{s} \zeta_{1}\right) u\right\|_{\epsilon}^{2}
\end{aligned}
$$

On the other hand, since $\left[\zeta_{1}, \log (\Lambda)\right] R^{s}$ has order 0 , then

$$
\begin{aligned}
&\left\|\left(\zeta_{1} \log (\Lambda) R^{s} \zeta_{1}\right) \bar{f}^{k} u\right\|_{\epsilon}^{2} \underset{\sim}{\sim} \|\left(\log (\Lambda)\left(\zeta_{1} R^{s} \zeta_{1}\right) \bar{f}^{k} u\left\|_{\epsilon}^{2}+\right\| \bar{f}^{k} u \|_{\epsilon}^{2}\right. \\
& \underset{\text { suplog estimate }}{<} \underbrace{\delta\left(\left\|L\left(\zeta_{1} R^{s} \zeta_{1}\right) \bar{f}^{k} u\right\|_{\epsilon}^{2}+\left\|\bar{L}\left(\zeta_{1} R^{s} \zeta_{1}\right) \bar{f}^{k} u\right\|_{\epsilon}^{2}\right)}_{\text {absorbed by the last line of }(2.10)}+\left\|\bar{f}^{k} u\right\|_{\epsilon}^{2},
\end{aligned}
$$

where we are using the equality $\left[\Lambda_{t}^{\epsilon}, L\right]=0$ as well as $\left[\Lambda^{\epsilon}, \log (\Lambda)\right]=0$. In the same way, using again (2.11), we estimate the central term in the last line 
of (2.7) which was left aside, that is,

$$
\left\|\bar{L}\left(\zeta_{1} R^{s} \zeta_{1}\right) u\right\|^{2} \lesssim\left\|\left(\zeta_{1} R^{s} \zeta_{1}\right) \bar{L} u\right\|^{2}+\|u\|^{2} .
$$

What remains, is to estimate the last term in the bottom of (2.10) (together with the error term $\left.\left\|\bar{f}^{k}\left(\zeta_{1} R^{s} \zeta_{1}\right) u\right\|_{\epsilon}^{2}\right)$. First, from Jacobi identity we get

$$
\left[\bar{L} \bar{f}^{k}, \zeta_{1} R^{s} \zeta_{1}\right] \sim(0 \text {-order }) \bar{f}^{k}+\zeta_{1} \log (\Lambda) R^{s} \zeta_{1} \bar{f}^{k}+(0 \text {-order }) \bar{f}^{k}
$$

so that we are eventually reduced to estimate $\left\|\left(\zeta_{1} R^{s} \zeta_{1}\right) \bar{L} \bar{f}^{k} u\right\|^{2}$. This is the most difficult operation. We have (by the identity $\left[\bar{L}, \bar{f}^{k}\right] \sim \bar{f}^{k-1}$ )

$$
\left\|\left(\zeta_{1} R^{s} \zeta_{1}\right) \bar{L} \bar{f}^{k} u\right\|_{\epsilon}^{2} \lesssim \underbrace{\left\|\left(\zeta_{1} R^{s} \zeta_{1}\right) \bar{f}^{k} \bar{L} u\right\|_{\epsilon}^{2}}_{\text {good }}+\left\|\left(\zeta_{1} R^{s} \zeta_{1}\right) \bar{f}^{k-1} u\right\|_{\epsilon}^{2} .
$$

Next we estimate the last term in the line above which also serves as an estimate for the term $\left\|\bar{f}^{k}\left(\zeta_{1} R^{s} \zeta_{1}\right) u\right\|_{\epsilon}^{2}$ that was encountered before. We have

$$
\begin{aligned}
\underbrace{\left\|\left(\zeta_{1} R^{s} \zeta_{1}\right) \bar{f}^{k-1} u\right\|_{\epsilon}^{2}}_{(c)} & \sim(\underbrace{\left(\zeta_{1} R^{s} \zeta_{1}\right) \bar{f}^{k-1} u}_{*},\left(\zeta_{1} R^{s} \zeta_{1}\right)\left[\bar{L}, \bar{f}^{k}\right] u)_{\epsilon} \\
& =-\left(*,\left(\zeta_{1} R^{s} \zeta_{1}\right) \bar{f}^{k} \bar{L} u\right)_{\epsilon}+\left(*,\left(\zeta_{1} R^{s} \zeta_{1}\right) \bar{L}^{k} u\right)_{\epsilon} .
\end{aligned}
$$

Now,

$$
\left\{\begin{aligned}
\left|\left(*,\left(\zeta_{1} R^{s} \zeta_{1}\right) \bar{f}^{k} \bar{L} u\right)_{\epsilon}\right| & <s c\|*\|_{\epsilon}^{2}+\underbrace{\left\|\left(\zeta_{1} R^{s} \zeta_{1}\right) \bar{f}^{k} \bar{L} u\right\|_{\epsilon}^{2}}_{\text {good }} \\
\left|\left(*,\left(\zeta_{1} R^{s} \zeta_{1}\right) \bar{L} \bar{f}^{k} u\right)_{\epsilon}\right| & <\left|(\underbrace{\left(\left(\zeta_{1} R^{s} \zeta_{1}\right) \bar{f}^{k-1} f L u\right.}_{\text {good }} \underbrace{\left(\zeta_{1} R^{s} \zeta_{1}\right) \bar{f}^{k-1} u}_{\text {absorbed by }(\mathrm{c})})_{\epsilon}\right| \\
& +2\left|(\underbrace{*}_{\text {absorbed by }(\mathrm{c})}, \underbrace{\left[\bar{L},\left(\zeta_{1} R^{s} \zeta_{1}\right)\right] \bar{f}^{k} u}_{(d)})_{\epsilon}\right|
\end{aligned}\right.
$$

We estimate $(d)$. We notice that

$$
\left[\bar{L},\left(\zeta_{1} R^{s} \zeta_{1}\right)\right] \sim \zeta_{1} \log (\Lambda) R^{s} \zeta_{1}+(0 \text {-order })
$$

We also remark that

$$
\left\{\begin{array}{l}
{\left[\Lambda^{\epsilon} \zeta_{1}, \log (\Lambda)\right] R^{s} \text { has order } 0} \\
{\left[\zeta_{1}, \Lambda^{\epsilon}\right] R^{s} \text { has order } 0} \\
{\left[L, \Lambda^{\epsilon}\right]=0 \quad(i i i)}
\end{array}\right.
$$


Hence

$$
\|(d)\|_{\epsilon}^{2} \underset{\text { by } \underset{(2.12)}{<}}{<}\left\|\left(\zeta_{1} \log (\Lambda) R^{s} \zeta_{1}\right) \bar{f}^{k} u\right\|_{\epsilon}^{2}+\left\|\bar{f}^{k-1} u\right\|_{\epsilon}^{2}+\|u\|_{0}^{2}
$$

$$
\begin{gathered}
\underset{\text { by }(2.13)(\mathrm{i}) \text { and (ii) }}{\leq}\left\|\left(\log (\Lambda) \zeta_{1} \Lambda^{\epsilon} R^{s} \zeta_{1}\right) \bar{f}^{k} u\right\|_{0}^{2}+\left\|\bar{f}^{k-1} u\right\|_{\epsilon}^{2}+\|u\|_{0}^{2} \\
\underset{\text { by suplog estimate }}{\leq} \delta\left(\left\|L\left(\zeta_{1} \Lambda^{\epsilon} R^{s} \zeta_{1}\right) \bar{f}^{k} u\right\|^{2}+\left\|\bar{L}\left(\zeta_{1} \Lambda^{\epsilon} R^{s} \zeta_{1}\right) \bar{f}^{k} u\right\|^{2}\right) \\
+\underbrace{c_{\delta}\left\|\left(\zeta_{1} \Lambda^{\epsilon} R^{s} \zeta_{1}\right) \bar{f}^{k} u\right\|^{2}}_{\text {absorbed by (c) }}+\left\|\bar{f}^{k-1} u\right\|_{\epsilon}^{2}+\|u\|_{0}^{2},
\end{gathered}
$$

where the last absorption occurs because $\bar{f}^{k}=s c \bar{f}^{k-1}$.

Finally, the term with $\delta$ is absorbed by the last term in (2.10) (after we transform $\Lambda^{\epsilon}$ into $\|\cdot\|_{\epsilon}$ to fit into (2.10) and use the fact that $\left[\bar{L} \zeta_{1}, \Lambda^{\epsilon}\right] \sim$ $\dot{\zeta}_{1} \Lambda^{\epsilon-1} \bar{L}$ and $\left.\left[L \zeta_{1}, \Lambda^{\epsilon}\right] \sim \dot{\zeta}_{1} \Lambda^{\epsilon-1} L\right)$. This concludes the proof of (1.3).

Proof of Proposition 1.3. As above, we stay in the positive microlocal cone, the support of $\psi^{+}$, and consider only derivatives and cut-off with respect to $t$. From the trivial identity $[L, f] \sim 1$, and from $\left[L, \zeta_{0}\right] \sim \dot{\zeta}_{0} g_{1}$, we get

$$
\begin{aligned}
\left\|\zeta_{0} u\right\|_{s}^{2} & =\left([L, f] \zeta_{0} u, \zeta_{0} u\right)_{s} \\
& <\left\|\bar{f} \zeta_{0} \bar{L} u\right\|_{s}^{2}+\left\|\bar{f} \zeta_{0} L u\right\|_{s}^{2}+\left\|\bar{f} g_{1} \zeta_{1} u\right\|_{s}^{2}+s c\left\|\zeta_{0} u\right\|_{s}^{2}
\end{aligned}
$$

Now, the last term is absorbed. As for the term before

$$
\begin{aligned}
& \left\|\bar{f} g_{1} \zeta_{1} u\right\|_{s}^{2} \underset{\text { by }}{\leq}\left\|\bar{f} g_{1 \overline{1}}^{\frac{1}{2}} \Lambda^{\frac{1}{2}} \zeta_{1} u\right\|_{s-\frac{1}{2}}^{2} \\
& \underset{\text { by }}{\leq}\left\|\bar{f} L \zeta_{1} u\right\|_{s-\frac{1}{2}}^{2}+\left\|\bar{f} \bar{L} \zeta_{1} u\right\|_{s-\frac{1}{2}}^{2}+\left\|\zeta_{1} u\right\|_{s-\frac{1}{2}}^{2} \\
& \lesssim\left\|\zeta_{1} \bar{f} L u\right\|_{s-\frac{1}{2}}^{2}+\left\|\bar{f} \bar{L} \zeta_{1} u\right\|_{s-\frac{1}{2}}^{2}+\left\|\bar{f} \zeta_{2} u\right\|_{s-\frac{1}{2}}^{2}+\left\|\zeta_{1} u\right\|_{s-\frac{1}{2}}^{2},
\end{aligned}
$$

for $\zeta_{2} \succ \zeta_{1}$. Now, $\left\|\bar{f} \zeta_{2} u\right\|_{s-\frac{1}{2}}^{2}$ and $\left\|\zeta_{1} u\right\|_{s-\frac{1}{2}}^{2}$ are not absorbable by $\left\|\zeta_{0} u\right\|_{s}^{2}$, but can be estimated by the 0 -norm using induction over $j$ such that $\frac{j}{2} \geq s$.

Proof of Proposition 1.4. As always, we stay in the positive microlocal cone and take derivatives and cut-off only in $t$. We prove the result for $s$ replaced by 0 and $\epsilon$ replaced by $-\eta$. The conclusion for general $s$ follows from the 
fact that $\partial_{t}$ commutes with $L$ and $\bar{L}$. We define

$$
v_{\lambda}=e^{-\lambda\left(e^{-\frac{1}{|z|^{\alpha}}}-i t+\left(e^{-\frac{1}{|z| \alpha}}-i t\right)^{2}\right)} \quad \lambda>>0 .
$$

We denote by $-\lambda A$ the term at exponent and note that $\operatorname{Re} \lambda A \sim \lambda\left(e^{-\frac{1}{|z|^{\alpha}}}+\right.$ $t^{2}$ ). For $L=\partial_{z}+i g_{1}(z) \partial_{t}$, we have $\bar{L} v_{\lambda}=0$ (which is the key point) and moreover, since $|\bar{f}|^{k} \sim|z|^{k}$

$$
\left|\bar{f}^{k} L v_{\lambda}\right| \sim \lambda|z|^{k-(\alpha+1)} e^{-\lambda\left(e^{-\frac{1}{|z|^{\alpha}}}+t^{2}\right)} e^{-\frac{1}{|z|^{\alpha}}}
$$

We set

$$
\lambda\left(e^{-\frac{1}{|z|^{\alpha}}}, t\right)=\left(\theta_{1}, \frac{1}{\sqrt{\lambda}} \theta_{2}\right) .
$$

Under this change we have, over $\operatorname{supp} \zeta_{0}$ and $\operatorname{supp} \zeta_{1}$ which implies $\theta_{1}<<\lambda$,

$$
|z|^{k-(\alpha+1)}=\frac{1}{\left(\log \lambda-\log \theta_{1}\right)^{\frac{k-(\alpha+1)}{\alpha}}} .
$$

Hence we interchange

$$
\left|\bar{f}^{k} L v_{\lambda}\right| \rightarrow \frac{1}{(\log \lambda)^{\frac{k-(\alpha+1)}{\alpha}}}\left(\frac{\theta_{1}+\theta_{2}^{2}}{\left(1-\frac{\log \theta_{1}}{\log \lambda}\right)^{\frac{k-(\alpha+1)}{\alpha}}}\right) e^{-\left(\theta_{1}+\theta_{2}^{2}\right)}
$$

Notice that $\theta_{1}<<\lambda$ and hence, for suitable positive $c_{1}$ and $c_{2}$, we have $c_{1}<\frac{\theta_{1}+\theta_{2}^{2}}{\left(1-\frac{\log \theta_{1}}{\log \lambda}\right)^{\frac{k-(\alpha+1)}{\alpha}}}<c_{2}$, uniformly over $\lambda$. We also interchange

$$
v_{\lambda}-\rightarrow e^{-\left(\theta_{1}+\theta_{2}^{2}\right)}
$$

Taking $L^{2}$ norms yields

$$
\left\|\bar{f}^{k} L v_{\lambda}\right\|^{2} \sim \frac{1}{(\log \lambda)^{2 \frac{k-(\alpha+1)}{\alpha}}}\left\|v_{\lambda}\right\|^{2}
$$

So, the effect on $L^{2}$ norm of the action of $\bar{f}^{k} L$ over $v_{\lambda}$ is comparable to $\frac{1}{(\log \lambda)^{\frac{k-(\alpha+1)}{\alpha}}}$. We describe now the effect of the pseudodifferential operator 
$\log \left(\Lambda_{t}\right)$. We claim that

$$
\left\|\log \left(\Lambda_{t}\right) e^{-\lambda t^{2}}\right\|^{2} \sim(\log \lambda)^{2}\left\|e^{-\lambda t^{2}}\right\|^{2}
$$

This is a consequence of

$$
\log \left(\Lambda_{t}\right) e^{-\lambda t^{2}} \sim \log \lambda e^{-\lambda t^{2}}+\left.\left(\log \left(\Lambda_{\tilde{t}}\right) e^{-\tilde{t}^{2}}\right)\right|_{\tilde{t}=\sqrt{\lambda} t}
$$

that we go to prove now. Using the coordinate change $\tilde{\theta}=\sqrt{\lambda} \theta, \tilde{\xi}=\frac{\xi}{\sqrt{\lambda}}$, we get

$$
\begin{aligned}
\int e^{i t \xi} \log \left(\Lambda_{\xi}\right) & \left(\int e^{-i \xi \theta} e^{-\lambda \theta^{2}} d \theta\right) d \xi \\
& =\int e^{i t \sqrt{\lambda} \tilde{\xi}}\left(\log \left(\frac{1}{\lambda}+|\tilde{\xi}|^{2}\right)^{\frac{1}{2}}+\log (\sqrt{\lambda})\right)\left(\int e^{i \tilde{\xi} \tilde{\theta}-\tilde{\theta}^{2}} d \tilde{\theta}\right) d \tilde{\xi} \\
& =\log (\sqrt{\lambda}) e^{-\lambda t^{2}}+\left.\left(\log \left(\Lambda_{\tilde{t}}^{\lambda}\right) e^{-\tilde{t}^{2}}\right)\right|_{\tilde{t}=\sqrt{\lambda} t}
\end{aligned}
$$

where $\log \left(\Lambda_{\tilde{t}}^{\lambda}\right)$ is the operator with symbol $\log \left(\frac{1}{\lambda}+|\tilde{\xi}|^{2}\right)^{\frac{1}{2}}$. This proves (2.16) and in turn the claim (2.15). In the same way, we can check that $\left\|\Lambda_{t}^{-\eta} e^{-\lambda t^{2}}\right\|^{2} \sim \lambda^{-2 \eta}\left\|e^{-\lambda t^{2}}\right\|^{2}$.

We combine now the effect over $v_{\lambda}$ of $\bar{f}^{k} L$ with that of $\log \left(\Lambda_{t}\right)$. If

$$
\left\|\zeta_{0} v_{\lambda}\right\|^{2}<\left\|\zeta_{1}\left(\log \Lambda_{t}\right)^{r} \bar{f}^{k} L v_{\lambda}\right\|^{2}+\left\|v_{\lambda}\right\|_{-\eta}^{2}
$$

then, since the right side is estimated from above by

$$
\left((\log \lambda)^{2 r}(\log \lambda)^{-2 \frac{k-(\alpha+1)}{\alpha}}+\lambda^{-2 \eta}\right)\left\|v_{\lambda}\right\|^{2}
$$

we must have that the logarithmic term is not infinitesimal which forces $r \geq \frac{k-(\alpha+1)}{\alpha}$.

\section{References}

[1] A. Bove, M. Derridj, J.J. Kohn and D.S. Tartakoff-Sums of squares of complex vector fields and (analytic-) hypoellipticity, Math. Res. Lett. 13 n.5 (2006), 683-701 
[2] D. Bell and S. Mohammed-An extension of Hörmander thorem for infinitely degenerate second-order operators, Duke Math. J. 78 (1995), 453-475

[3] M. Christ-Hypoellipticity of the Kohn Laplacian for threedimensional tubular Cauchy-Riemann structures, J. of the Inst. of Math. Jussieu 1 (2002), 279-291

[4] G.B. Folland and J.J. Kohn-The Neumann problem for the Cauchy-Riemann complex, Ann. Math. Studies, Princeton Univ. Press, Princeton N.J. 75 (1972)

[5] L. Hörmander-Hypoelliptic second order differential equations, Acta Math. 119 (1967), 147-171

[6] J.J. Kohn-Hypoellipticity at points of infinite type, Contemporary Math. 251 (2000), 393-398

[7] J.J. Kohn-Superlogarithmic estimates on pseudoconvex domains and CR manifolds, Annals of Math. 156 (2002), 213-248

[8] J.J. Kohn-Hypoellipticity and loss of derivatives, Annals of Math. 162 (2005), 943-986

[9] J.J. Kohn and L. Nirenberg-Non-coercive boundary value problems, Comm. Pure Appl. Math. 18 (1965), 443-492

[10] S. Kusuoka and D. Stroock - Applications of Mallavain calculus II, J. Fac. Sci. Univ. Tokyo Sec. IA Math. 32 (1985), 1-76

[11] T.V. Khanh, S. Pinton and G. Zampieri-Loss of derivatives for systems of complex vector fields and sums of squares, Proc. of the AMS 140 n. 2 (2012), 519-530

[12] T.V. Khanh and G. Zampieri-Regularity of the $\bar{\partial}$-Neumann problem at a point of infinite type, J. Funct. Analysis 255 (2010), 2760-2775

[13] Y. Morimoto-Hypoellipticity for infinitely degenerate elliptic operators, Osaka J. MAth. 24 (1987), 13-35

[14] E.M. Stein - An example on the Heisenberg group related to the Lewy operator, Invent. Math. 69 (1982), 209-216 
Tran Vu Khanh

School of Mathematics and Applied Statistics,

University of Wollongong,

NSW, Australia, 2522

E-mail: tkhanh@uow.edu.au

Stefano Pinton

Dipartimento di Matematica,

Università di Padova,

via Trieste 63, 35121 Padova, Italy

E-mail: pinton@math.unipd.it

Giuseppe Zampieri

Dipartimento di Matematica,

Università di Padova,

via Trieste 63, 35121 Padova, Italy 
